\title{
Erratum to: Bionic Optimization Design of Electronic Nose Chamber for Oil and Gas Detection
}

\author{
Zhiyong Chang ${ }^{1,2,3}$, Youhong Sun ${ }^{3,4^{*}}$, Yuchen Zhang ${ }^{1,2}$, Yanli Gao ${ }^{5}$, Xiaohui Weng ${ }^{2,6}$, \\ Donghui Chen ${ }^{1,2}$, Liewe David ${ }^{7}$, Jun Xie ${ }^{1,2,8}$ \\ 1. College of Biological and Agricultural Engineering, Jilin University, Changchun 130022, China \\ 2. Key Laboratory of Bionic Engineering, Ministry of Education, Jilin University, Changchun 130022, China \\ 3. National-Local Joint Engineering Laboratory of In-situ Conversion, Drilling and Exploitation Technology for Oil Shale, \\ Jilin University, Changchun 130021, China \\ 4. College of Construction Engineering, Jilin University, Changchun 130022, China \\ 5. Clinical Medicine, Bethune First Hospital of Jilin University, Changchun 130021, China \\ 6. College of Mechanical Science and Engineering, Jilin University, Changchun 130022, China \\ 7. School of Computing and Technology, the University of Gloucestershire, the Park, Cheltenham GL50 2RH, UK \\ 8. Air Combat Service Academy, Air Force Aviation University, Changchun 130021, China
}

Copyright (C) 2018, Jilin University.

Erratum to: J Bionic Eng (2018) 15(3): 533-544

https://doi.org/10.1007/s42235-018-0044-6

The first affiliation of the first author was incorrect in the published paper and should be revised as "1. College of Biological and Agricultural Engineering, Jilin University, Changchun 130022, China". 